\title{
Efficacy and safety of caspofungin in children: Systematic review and meta-analysis
}

\author{
María T. Rosanova, M.D., Ph.D. ${ }^{a}$, David Bes, M.D. ${ }^{b}$, Pedro Serrano Aguilar, M.D., Ph.D. ${ }^{c}$, \\ Leticia Cuellar Pompa, M.D. ${ }^{d}$, Norma Sberna, Pharmacist ${ }^{e}$ and Roberto Lede, M.D., Ph.D. ${ }^{f}$
}

a. Department of Epidemiological Control and Infectology of Hospital "Prof. Dr. J. P. Garrahan", Buenos Aires, Argentina.

b. Department of General Medicine of Hospital “Prof. Dr. J. P. Garrahan", Buenos Aires, Argentina.

c. Evaluation Unit of the Canary Islands Health Service (SESCS), National Network of Health Services Research in Chronic Diseases (REDISSEC), Tenerife, Spain.

d. Canary Islands Foundation of Health Care Research (FUNCANIS), Tenerife, Spain.

e. Pharmacy Department of Hospital "Prof. Dr. J. P. Garrahan", Buenos Aires, Argentina.

f. Master in Clinical Pharmacology Research (CONEAU. Resolution 416/11) of Universidad Abierta Interamericana (UAI), Buenos Aires, Argentina.

E-mail address:

María T. Rosanova, M.D.: margris2@yahoo.com.ar

Funding: None.

Conflict of interest: None.

Received: 12- 2-2015

Accepted: 2-22-2016

\section{ABSTRACT}

Invasive fungal infections are a significant cause of morbidity and mortality in children. Caspofungin is an echinocandin used as an alternative treatment in the prevention and/or treatment of certain invasive fungal infections in children, although compared to the standard treatment there is little evidence on its efficacy and safety.

Objectives. To evaluate the efficacy and safety of caspofungin compared with other antifungal drugs for the prevention and/or treatment of invasive fungal infections in children.

Material and methods. The objective of the initial search strategy was to identify randomized controlled studies of acceptable methodological quality (Jadad scale $>3$ ), through the key word "caspofungin", conducted in patients with an age range from 0 to 18 years old.

Results. Only 3 publications met the inclusion criteria. Two of them were studies conducted in children and one in newborn infants. A higher incidence of adverse events was not documented for caspofungin and its efficacy was not different from that of other antifungal drugs (typical RR 1.47; CI 95\%: 0.78-2.79).

Conclusions. This systematic review suggests that caspofungin could be considered as an alternative drug in children for the prevention and treatment of invasive fungal infections.

However, given the small number of existing publications, more studies are required to reach definite conclusions.

Key words: caspofungin, mycosis, efficacy, child, safety.

http:/ /dx.doi.org/10.5546/aap.2016.eng.305

\section{INTRODUCTION}

Invasive fungal infections (IFI) are a significant cause of morbidity and mortality in children. ${ }^{1}$ The presence of neutropenia secondary to chemotherapy or to bone marrow transplantation, low birth weight and prematurity, among others, are risk factors for fungal infections. In these cases, amphotericin B deoxycholate and its different presentations are the most widely used treatment agents. Its use limits the possible associated adverse events like nephrotoxicity. Other formulations of amphotericin are much more expensive and the azole group presents drug interactions; therefore, having treatment alternatives would be useful in pediatrics.

Caspofungin (ATC: J02AX04) is an antifungal agent of the echinocandin family and has a clear spectrum of action against Candida spp. and Aspergillus spp. It works by inhibiting the synthesis of $(1,3)$-D-glucan which is a component of fungus cell wall, and since it is not present in mammal cell wall it would account for the small incidence of adverse events. It has been shown that caspofungin is effective in esophageal and in invasive candidiasis, as rescue therapy in aspergillosis and as empirical therapy of neutropenia and fever in adults. ${ }^{5}$ It is important to point out that its resistance potential is low. Its apparent low toxicity and the lack of interactions make caspofungin a useful alternative in fungal infection prophylaxis and treatment. In spite of having been approved by the Food and Drug Administration (FDA) for its use in children aged 3 months and older, the experience in children is still limited. ${ }^{4,5}$

The need to have treatment alternatives available, of proven effectiveness and safety, to manage IFIs among children, and the limited experience with the use of caspofungin in our setting, explains the objective of this study, in which a systematic review about caspofungin effectiveness and safety was conducted, in comparison with standard antifungal agents, to manage the prophylaxis and/or treatment of proven, probable or possible IFIs in children. 


\section{Procedures}

a) Bibliographic search

A bibliographic search was done combining the endpoint "randomized controlled studies" with the key word "caspofungin" and the 0 to 18 year age filter was added to the search. the strategy was adapted from the following databases: Medline and PreMedline (OivdSP interface) from 1966 to August 2015; EMBASE (Elsevier interface) from 1974 to August 2015; Cumulative Index to the Nursing and Allied Health Literature (CINAHL) (EbscoHost interface) from 1982 to August 2015; Sciences Citation Index Expanded (SCI-EXPANDED) (Web of Science interface) from 1990 to August 2015; Cochrane Library (Wiley interface) from 1989 to August 2015; Scopus (Elsevier interface) from 1960 to August 2015; and DARE, HTA (CRD interface) from 1960 to August 2015. No language restriction was applied.

\section{b) Study selection criteria}

Only randomized, controlled studies comparing the effectiveness and safety of caspofungin with those of any other antifungal agents, for the prophylaxis and/or treatment in children were included. The primary endpoint was efficacy, defined as the absence of clinical signs-symptoms and/or culture negativization in case they were positive. The incidence of adverse events was also evaluated. Two independent reviewers (MTR and DB) selected articles by title and abstract, and analyzed the complete text of those meeting inclusion criteria. A third reviewer (RL) participated in case of disagreement.

\section{c) Narrative and quantitative evaluation and synthesis}

Each included study was evaluated; characteristics of each of them were extracted (design, participants, interventions, results) and the bias control was estimated by means of the Jadad scale. ${ }^{6}$ For the quantitative synthesis, a relative risk indicator was used with $95 \%$ confidence intervals obtained by the randomized effect model (Der Simonian-Laird). When the hypothesis test was used, the null hypothesis was rejected if $\mathrm{p}<0.05$. Heterogeneity among studies was measured with the I 2 index. Publication bias was determined with a funnel plot graph. The software MetaAnalyst (Truft Medical Center) was used.

\section{RESULTS}

The bibliographic search contributed with 841 articles of which 653 were subtracted after removing search duplications. After reviewing abstracts, 21 randomized and controlled studies of echinocandin were identified, therefore full texts were reviewed. Only 3 of them met inclusion criteria (Figure 1).

Table 1 presents a summary of the most relevant characteristics describing the 3 selected studies.

\section{Description of the included studies:}

1) Maertens et al., ${ }^{4}$ assessed, in a multicentric, double blind study, caspofungin treatment in 2-17 year old patients undergoing chemotherapy or bone marrow transplantation after 4 or more days of febrile neutropenia. Patients were randomly assigned to receiving caspofungin at a loading dose of $70 \mathrm{mg} / \mathrm{m}^{2}$, followed by a daily dose of $50 \mathrm{mg} / \mathrm{m}^{2}$ plus placebo or $3 \mathrm{mg} / \mathrm{kg} /$ day of liposomal amphotericin plus placebo. The efficacy was evaluated based on the rate of successful treatments of baseline fungal infections, or the absence of a breakthrough infection, or the absence of a fungal infection within 7 days after completion of treatment, and survival after 7 days of treatment. Withdrawal from protocol due to drug-related toxicity or lack of efficacy, and fever resolution within the first 48 hours of treatment were also studied. Eighty three patients were included in the study; 81 out of them could be analyzed on an intention-totreat basis. A favorable overall response was evidenced in $46.4 \%(26 / 56)$ of patients assigned to the caspofungin arm and in $32 \%(8 / 25)$ of those treated with liposomal amphotericin. In higher risk patients, like those who had undergone a bone marrow transplantation or who had relapsing leukemia, the response was favorable in $9 / 15(60 \%)$ of the patients treated with caspofungin and in none of the 7 patients treated with liposomal amphotericin ( $p>0.05)$. No deaths were reported in either of the two groups up to 7 days after having completed treatment. When fungal infection was suspected, fever resolution was observed in $24 / 56$ children (43\%) treated with caspofungin and in $8 / 25(32 \%)$ treated with liposomal amphotericin $(\mathrm{p}>0.05)$. Drug discontinuation rate because of adverse events was $4 \%$ in the group treated with caspofungin vs. $12 \%$ in the group treated with amphotericin ( $p>0.05)$. In this study, the likelihood of biases is low (Jadad 5). 
2) In a randomized, double blind study, Mohamed et al. evaluated the efficacy and safety of capsofungin compared to amphotericin B deoxycholate in 32 newborn infants with microbiological confirmation of invasive Candida spp. infection. ${ }^{7}$ The study consisted in the administration of capsofungin $2 \mathrm{mg} / \mathrm{kg} /$ day and amphotericin B $1 \mathrm{mg} / \mathrm{kg} /$ day during, at least, 14 days. Efficacy was evaluated based on 5 items: fungal infection clinical resolution, microbiological eradication, absence of breakthrough infection considered since treatment onset up to 7 days of having completed it, survival rate after 7 days of treatment completion and treatment discontinuation due to drug related toxicity or lack of efficacy. Clinical adverse events and laboratory abnormalities were evaluated. Clinical adverse events included infusion related compromise while among laboratory abnormalities the most frequent were liver and kidney toxicities (serum creatinine duplication) and hypokalemia requiring potassium supplementation. Microbiological culture evidenced C. albicans: $75 \%$; C. parapsilosis: 15.6\%; C. tropicales: $9.4 \%$. Compared efficacy of treatments reached $86.7 \%$ among patients treated with capsofungin and $41.7 \%$ among those treated with amphotericin $B(p=0.04)$. Clinical adverse events and laboratory abnormalities were lower in the group treated with capsofungin $(p<0.05)$. None of the patients treated with capsofungin required treatment withdrawal due to adverse events while $5(29.4 \%)$ patients treated with amphotericin B did require it. Mortality was similar between both groups ( 1 in caspofungin group vs. 3 in the amphotericin group, $\mathrm{p}=0.6$ ) and was not attributed to fungal therapy in any of the two groups.

Conclusions are that capsofungin is more effective and safe than amphotericin B deoxycholate for the treatment of neonatal invasive Candida spp. infections. Bias control

FIGURE 1. Bibliographic search and article selection flow chart

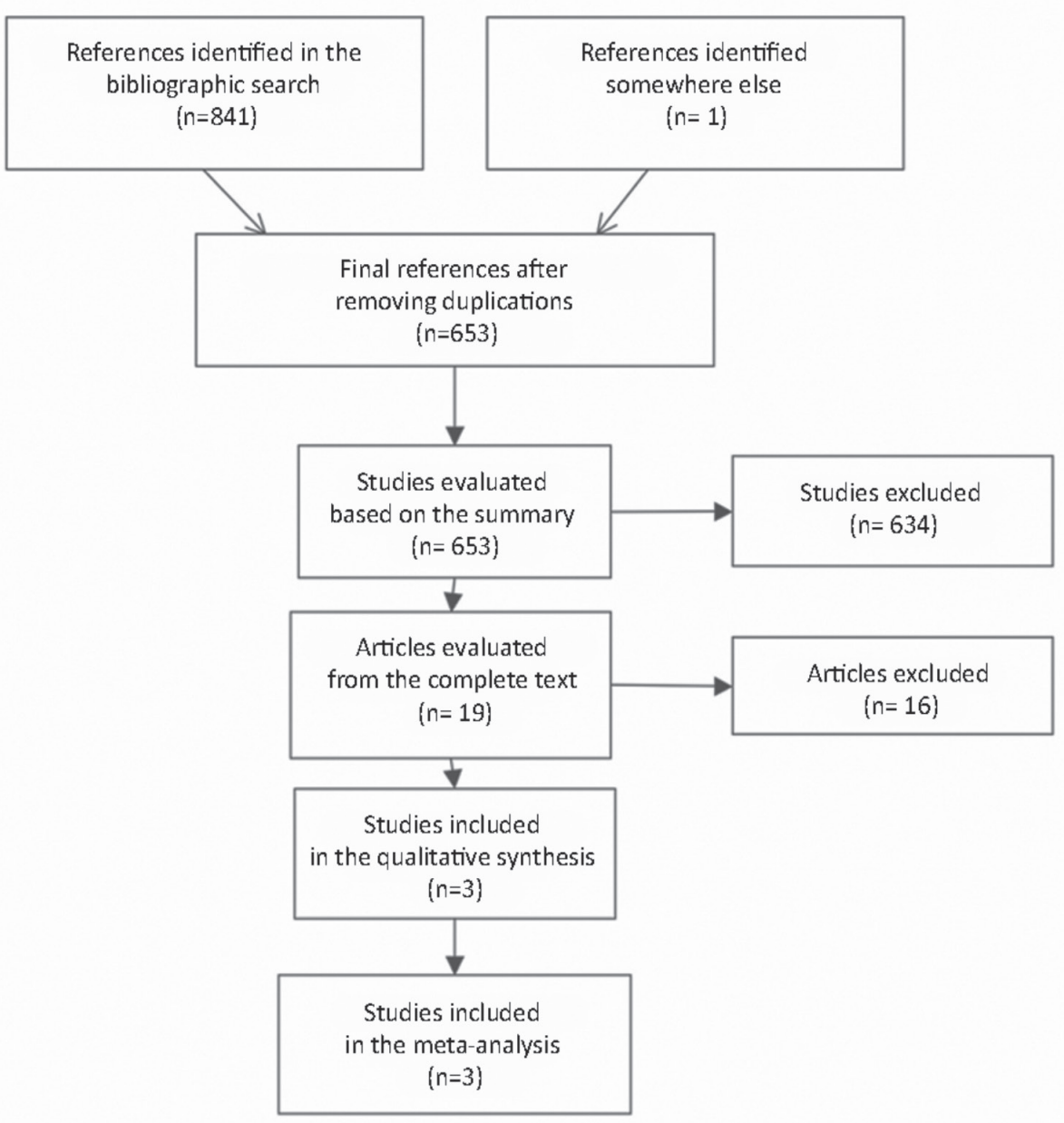


suggests that the likelihood of its presence is low (Jadad 5).

3) A multicentric and randomized study published by Caselli et al., ${ }^{5}$ analyzed 110 neutropenic children (undergoing chemotherapy or autologous bone marrow transplantation) with persistent fever on day 4 in spite of antibiotic treatment and, consequently, with risk of having an IFI. Follow-up was extended to at least 30 days. Six patients were excluded because of the diagnosis of IFI when enrolled in the study; therefore, the final comparative analysis included 104 patients. They were stratified into high $(n=56)$ and low risk $(n=48)$ of having IFI and also, by variables that reinforced group homogeneity. Those of low risk were randomized to receiving the following: group A, no antifungal agent; group B, liposomal

TABLE 1. Main characteristics of the included studies

\begin{tabular}{|c|c|c|c|c|c|}
\hline Study & Design & Patient & Intervention & $\begin{array}{l}\text { Evaluated } \\
\text { results }\end{array}$ & $\begin{array}{c}\text { Jadad } \\
\text { scale }\end{array}$ \\
\hline Maertens et al. ${ }^{4}$ & $\begin{array}{l}\text { Randomized, } \\
\text { controlled, } \\
\text { double blind. }\end{array}$ & $\begin{array}{l}\text { Eighty one } \\
2-17 \\
\text { pediatric } \\
\text { patients with } \\
\text { persistent } \\
\text { febrile } \\
\text { neutropenia. }\end{array}$ & $\begin{array}{l}\text { Capsofungin: } \\
\text { loading dose of } \\
70 \mathrm{mg} / \mathrm{m}^{2} \text { and } \\
\text { then } \\
50 \mathrm{mg} / \mathrm{m}^{2} / \text { day, } \\
\text { or amphotericin, } \\
\mathrm{L}-\mathrm{AmB} \text { : } \\
3 \mathrm{mg} / \mathrm{kg} / \text { day } \\
\text { in } 2: 1 . \\
\text { Doses could be } \\
\text { increased if the } \\
\text { clinical status } \\
\text { worsened. }\end{array}$ & $\begin{array}{l}\text { Themain objective was to estimate caspofungin } \\
\text { and L-AmB safety, tolerance and efficacy. } \\
\text { Safety: the rate of patients with } 1 \text { or moreclinical } \\
\text { adverse events or laboratory abnormalities } \\
\text { were evaluated during the treatment period } \\
\text { and } 14 \text { days after. } \\
\text { Efficacy was based on the evaluation of the } \\
\text { overall fulfillment of } 5 \text { criteria: symptom } \\
\text { resolution, culture negativization, absence } \\
\text { of fungal infection without treatment, } 7 \text { day } \\
\text { survivalafter finishing treatmentand treatment } \\
\text { discontinuation because of adverse events. }\end{array}$ & 5 \\
\hline Mohamed et al. ${ }^{7}$ & $\begin{array}{l}\text { Prospective, } \\
\text { randomized, } \\
\text { controlled, } \\
\text { double-blind }\end{array}$ & $\begin{array}{l}\text { Thirty two } \\
\text { newborn } \\
\text { infants } \\
\text { with the } \\
\text { diagnosis } \\
\text { of invasive } \\
\text { Candida } \\
\text { infection, } \\
\text { confirmed by } \\
\text { CSF, blood } \\
\text { or urine } \\
\text { culture. }\end{array}$ & $\begin{array}{l}\text { Capsofungin } \\
\text { ( } 2 \mathrm{mg} / \mathrm{kg} / \text { day) } \\
\text { or amphotericin: } \\
\text { amphotericin B } \\
\text { deoxycholate } \\
(1 \mathrm{mg} / \mathrm{kg} / \text { day) } \\
\text { by IV route, } \\
\text { during }>1 \mathrm{~h} \text {. } \\
\text {. }\end{array}$ & $\begin{array}{l}\text { Efficacy and safety were evaluated. } \\
\text { Safety and tolerance were evaluated in terms } \\
\text { of clinical events and laboratory abnormalities. } \\
\text { Efficacy was evaluated in terms of the response } \\
\text { based on the overall fulfillment of } 5 \text { criteria: } \\
\text { symptom resolution, culture negativization, } \\
\text { absence of fungal infection without treatment, } \\
7 \text { day survival after finishing treatment and } \\
\text { treatment discontinuation because of adverse } \\
\text { events. }\end{array}$ & 5 \\
\hline Caselli et al. ${ }^{5}$ & $\begin{array}{l}\text { Randomized, } \\
\text { controlled, } \\
\text { stratified. } \\
\text { No double } \\
\text { blind. }\end{array}$ & $\begin{array}{l}\text { One hundred } \\
\text { and four } \\
\text { pediatric } \\
\text { patients } \\
\text { younger } \\
\text { than } 18 \\
\text { years of age, } \\
\text { with febrile } \\
\text { neutropenia } \\
\text { after } 4 \text { days } \\
\text { of antibiotic } \\
\text { treatment. }\end{array}$ & $\begin{array}{l}\text { High risk } \\
\text { patients were } \\
\text { randomized } \\
\text { to receiving } \\
\text { treatment with } \\
\text { B) L-Amb or } \\
\text { C) caspofungin. } \\
\text { The low risk } \\
\text { group was } \\
\text { randomized } \\
\text { to A) without } \\
\text { treatment, } \\
\text { B) L-Amb, } \\
\text { C) capsofungin. }\end{array}$ & $\begin{array}{l}\text { Drug toxicity was evaluated taking into account } \\
\text { kidney or liver toxicity development defined } \\
\text { in the exclusion criteria. } \\
\text { The efficacy was evaluated in terms of the } \\
\text { response based on the overall fulfillment } \\
\text { of } 3 \text { criteria. }\end{array}$ & 3 \\
\hline
\end{tabular}

L-AmB: liposomal amphotericin; CSF: cerebrospinal fluid. 
amphotericin B; and group C, caspofungin at the same doses as those used in high risk patients. Besides antibiotic treatment, high risk patients were randomized to receiving: group $\mathrm{B}$, liposomal amphotericin B ( $3 \mathrm{mg} / \mathrm{kg} /$ day by IV route) and group C, caspofungin (a loading dose of $70 \mathrm{mg} / \mathrm{m}^{2}$ and then $50 \mathrm{mg} / \mathrm{m}^{2} /$ day). The primary objective was to evaluate the clinical efficacy and relevance of antifungal treatment among patients with febrile neutropenia and a low risk of fungal infection. The primary end-point was complete response to treatment defined by fever resolution $<37.5^{\circ} \mathrm{C}$ during $>48$ hours, 30-day survival without evidence of IFI and having completed the treatment assigned at randomization. Secondary objectives were to compare: a) liposomal amphotericin B and caspofungin efficacy in patients with febrile neutropenia and, b) the toxicological profile of both antifungal agents. Although the study was designed for a larger population, it had to be interrupted due to modifications in the rules regulating experimental studies in the country of origin and it was ended because of futility. ${ }^{8}$ Results showed that 6 patients initially had IFI and 3 developed an infection in the course of the study (9 patients, $8.2 \%$, CI 95\%: 3.8-15.0). A case of cryptococcosis was documented, and 4 cases of probable Aspergilus spp. infection and 4 cases of possible Aspergilus spp. infection were diagnosed. No severe grade III-IV drug related toxicity was observed in any patients, while grade I-II drug related toxicity cases were evenly distributed among the three groups. Complete response to empirical antifungal treatment was reached in $90 / 104$ patients $(86.5 \%): 48 / 56$ of the high risk group $(85.7 \%)$ and $42 / 48(87 . \%)$ of the low risk group. In high risk patients, no significant differences were seen regarding complete response to treatment $-88 \%$ in arm b) and $83.9 \%$ in arm c) ( $p>0.05)$ - neither in hospital length of stay nor in fever duration. In low risk patients, there were no significant differences either as far as complete response to treatment $-87.5 \%$ in arm a), $80 \%$ in arm b) and $94.1 \%$ in arm c) $(\mathrm{p}=0.41)$ - neither in hospital length of stay nor in fever duration. Authors concluded that antifungal treatment did not seem to be necessary in patients with a low risk of developing IFI in terms of survival and fever persistence. High risk patients would be the group in the population who could benefit the most with an antifungal treatment. Both the compared efficacy as well as the incidence of adverse events between amphotericin and caspofungin were similar. In none of the patients cohorts, deaths were reported within 7 days after treatment completion.

The study had a moderate risk of biases (Jadad 3), because neither masking nor double blinding was possible.

\section{Quantitative synthesis:}

Table 2 presents data of each study and the summary measure. The direction of the effect of two of them (Maertens ${ }^{4}$ and Mohamed ${ }^{7}$ ) suggests that caspofungin is more effective than amphotericin. However, in the study of Caselli et al., ${ }^{5}$ the difference is almost null. The overall summary measure (RR 1.47) shows a direction of the effect favoring caspofungin, but without the necessary accuracy to be able to assert it (CI 95\%: $0.78-2.79)$. The set of studies was heterogeneous (I2: 0.77), that justified the analysis based on the random effect model. The sensitivity analysis, which only took into account high risk patients, resulted in a typical RR of 1.55, CI 95\%: 0.68-3.4, which is not considerably different from the one obtained in the overall analysis. The incidence of adverse events (Table 3) showed no heterogeneous results (I2: 0.19) and the summary measure (RR) was 1.17 , CI 95\%: 0.46-2.97.

\section{DISCUSSION}

The aim of this study is to summarize the best scientific knowledge currently available about caspofungin efficacy and safety in the management of IFI in children. ${ }^{1-4}$ This review is timely because it explains why the use of

TABLE 2. Efficacy data of each study, their individual RRs and abstract measures

\begin{tabular}{|c|c|c|c|c|c|c|}
\hline \multirow[t]{2}{*}{ Study } & \multicolumn{2}{|c|}{ Caspofungin } & \multicolumn{2}{|c|}{ Amphotericin } & \multirow[t]{2}{*}{ RR (CI 95\%) } & \multirow[t]{2}{*}{ Relative weight } \\
\hline & Cure & Total & Cure & Total & & \\
\hline Maertens, 2010 & 26 & 56 & 8 & 25 & $1.45(0.77-2.74)$ & 0.31 \\
\hline Mohamed, 2012 & 13 & 15 & 5 & 17 & $2.94(1.37-6.32)$ & 0.27 \\
\hline Caselli, 2012 & 26 & 31 & 22 & 25 & $0.95(0.77-1.18)$ & 0.42 \\
\hline
\end{tabular}

Typical RR (randomized model, Der Simonian-Laird): 1.47; 95\% confidence interval [CI]: 0.78-2.79.

Heterogeneity: $I^{2}: 0.77$. RR: relative risk. 
standard antifungal agents, like amphotericin $B$ deoxycholate, have not allowed to reduce mortality in IFIs and has also resulted in significant potential adverse events like nephrotoxicity. ${ }^{3-5}$ The results of this systematic review are obtained from the narrative and quantitative synthesis of only three primary studies of good quality.

In one of them, caspofungin was more effective than amphotericin; ${ }^{4}$ in another one, the direction of the effect favored caspofungin but without enough accuracy; ${ }^{7}$ while in the third one, the direction was almost null. ${ }^{5}$

The common summary measure keeps a direction of the effect in favor of caspofungin but with a significant uncertainty that does not allow to ascertain it.

The secondary analysis, by subsets, conducted on higher risk patients did not yield a different result; neither it did regarding drug-induced nephrotoxicity. Overall, caspofungin seems to have a favorable efficacy without increasing the risk of adverse events in comparison with other antifungal agents; however, the limited number of studies, limitations in their sample sizes, age differences of included patients and a certain heterogeneity in the choice of outcome measures point the need of conducting additional research to confirm our conclusions.

In spite of the above mentioned limitations, the internal validity of the included studies is strong. In short, two studies providing favorable results for caspofungin are characterized because their quality is high (maximum score in Jadad scale)..$^{4-7}$

Additionally, results published by other authors who use less robust, observational designs, reinforce our findings by supporting the possible value and effectiveness of caspofungin as well as by providing information regarding the incidence, types and magnitude of associated adverse events. Prospective and retrospective,

TABLE 3. Safety data (nephrotoxicity) of each study, their individual RRs and abstract measures

\begin{tabular}{lcccccc}
\hline \multirow{2}{*}{ Study } & \multicolumn{2}{c}{ Caspofungin } & \multicolumn{2}{c}{ Amphotericin } & \multirow{2}{*}{ RR (CI 95\%) } & \multirow{2}{*}{ Relative weight } \\
& Cure & Total & Cure & Total & & 0.09 \\
Maertens, 2010 & 0 & 15 & 2 & 17 & $0.22(0.12-4.35)$ & 0.24 \\
Mohamed, 2012 & 3 & 55 & 2 & 25 & $0.68(0.12-3.82)$ & 0.67 \\
Caselli, 2012 & 15 & 48 & 7 & 40 & $1.78(0.80-3.95)$ & \\
\hline
\end{tabular}

Typical RR (randomized model, Der Simonian-Laird):1.17; CI 95\%: 0.46-2.97.

Heterogeneity: $I^{2}: 0.19$. RR: relative risk.

FIGURE 2. Forest Plot graph of the comparative efficacy results of caspofungin vs. amphotericin

Relative Risk (CI 95\%)

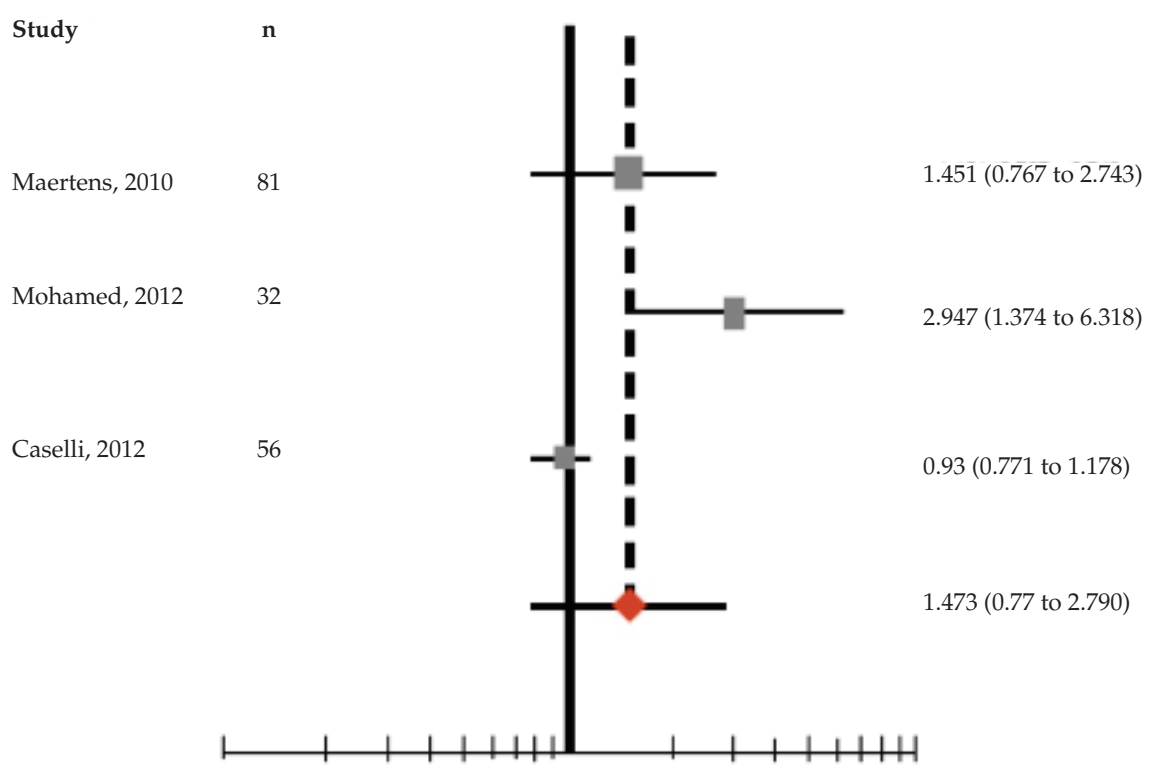


observational cohort studies are the most appropriate to document and characterize adverse events related to any type of technology assessment in health care (whether drug-related or not) once implemented. ${ }^{9}$

Zaoutis et al., in a prospective and multicentric cohort, describe the results in 49 children, with an age range from 3 months old to 17 years old, who received caspofungin as primary or rescue therapy due to documented or probable candidiasis or invasive aspergillosis, or documented esophageal candidiasis. They conclude that caspofungin is safe and effective. ${ }^{10}$

Odio et al., provide information about the possible therapeutic value of caspofungin in newborn infants with persistent candidemia treated with caspofungin combined with amphotericin in a lipid formulation, fluconazole or 5 fluorocytosine. ${ }^{11}$

One of the studies included in our review compared caspofungin to amphotericin B in newborn infants with confirmed invasive candidiasis. ${ }^{7}$ In this study, caspofungin was more effective and the incidence of adverse events was lower, results similar to the findings of other pediatric studies ${ }^{12-14}$ and those of Mora et al., who studied the use of this drug in adult patients with invasive candidiasis. ${ }^{15}$

At a higher level of evidence, the systematic review by Falagas et al., ${ }^{16}$ about adolescent and adult patients, in which, in agreement with our observations in the pediatric stage, they conclude that caspofungin could be an alternative agent in the treatment of invasive candidiasis y candidiasis esophagitis, since it has the same efficacy and less toxicity, without any changes in the mortality rate, as stated in other reports. ${ }^{17}$

The greatest contribution of this study is to be the first systematic review and meta-analysis on the efficacy of caspofungin in children and evidences the urgent need of conducting adequate studies with the necessary power to clearly respond to the question about the best antifungal agent to treat IFI in this population since the current evidence is modest and insufficient to ascertain the opinion. It is important not only because of the possibility of having a potentially useful agent and not using it (implicit risk in the low power of the studies) but also for using it even if it is not the best alternative. Let us not forget that we are dealing with the treatment of a severe condition in a particularly vulnerable population, like children with immune deficiency and newborn infants.

In order that the new pieces of evidence contribute to reducing current scientific uncertainty, participants enrollment and followup period should be improved in the studies while ensuring the validity of direct comparisons between caspofungin and other active antifungal agents habitually used in pediatrics.

\section{REFERENCES}

1. Blyth CC, Hale K, Palasanthiran P, O'Brien T, Bennett HM. Antifungal therapy in infants and children with proven, probable or suspected invasive fungal infections. Cochrane Database Syst Rev 2010;(2):CD006343.

2. McCormack PL, Perry C. Caspofungin A review of its use in the treatment of fungal infections. Drugs 2005;65(14): 2049-68.

3. Wiederhold NP, Herrera L. Caspofungin for the treatment of immunocompromised and severely ill children and neonates with invasive fungal infections. Clin Med Insights Pediatrics 2012;6:19-31.

4. Maertens JA, Madero L, Reilly AF, Lehrnbecher T, et al. A randomized, double-blind, multicenter study of caspofungin versus liposomal amphotericin B for empiric antifungal therapy in pediatric patients with persistent fever and neutropenia. Pediatr Infect Dis J 2010;29(5):415-20.

5. Caselli D, Cesaro S, Ziino O, Ragusa P, et al. A prospective, randomized study of empirical antifungal therapy for the treatment of chemotherapy-induced febrile neutropenia in children. Br J Haematol 2012;158(2):249-55.

6. Jadad AR, Moore RA, Carrol D; Jenkinson C, et al. Assessing the quality of reports of randomized clinical trials: is blinding necessary? Control Clin Trials 1996;17(1):1-12.

7. Mohamed WA, Ismail M. A randomized, double-blind, prospective study of caspofungin vs. amphotericin B for the treatment of invasive candidiasis in newborn infants. J Trop Pediatr 2012;58(1):25-30.

8. Freidlin B,KornEL. Acommentonfutility monitoring. Control Clin Trials 2002;23(4):355-66.

9. Von Elm E, Altman DG, Egger M, Pocock SJ, et al. Strengthening the Reporting of Observational Studies in Epidemiology (STROBE) Statement: Guidelines for reporting observational studies. BMJ 2007;335(7624):806-8.

10. Zaoutis T, Lehrnbecher T, Groll AH, Steinbach WJ, et al. Safety experience with caspofungin in pediatric patients. Pediatr Infect Dis J 2009;28(12):1132-5.

11. Odio C, Araya R, Pinto L, Castro CE, et al. Caspofungin therapy of neonates with invasive Candidiasis. Pediatr Infect Dis J 2004;23(12):1093-7.

12. Walsh TJ, Adamson PC, Seibel NL, Flynn PM, et al. Pharmacokinetics, safety, and tolerability of caspofungin in children and adolescents. Antimicrob Agents Chemother 2005;49(11):4536-45.

13. Caudle KE, Inger AG, Butler DR, Rogers PD. Echinocandin use in the neonatal intensive care unit. Ann Pharmacother 2012;46(1):108-16.

14. VandenBussche HL, Van Loo DA. A clinical review of echinocandins in pediatric patients. Ann Pharmacother 2010;44(1):166-77.

15. Mora-Duarte J, Betts R, Rotstein C, Colombo AL, et al. Comparison of caspofungin and amphotericin $\mathrm{B}$ for invasive candidiasis. N Engl J Med 2002;347(25):2020-9.

16. Falagas ME, Ntziora FA, BetsiGA,Samonis G. Caspofungin for the treatment of fungal infections: a systematic review of randomized controlled trials. Int J Antimicrob Agents 2007;29(2):136-43.

17. Yuan X, Wang R, Bai CQ, Song XJ, Liu YN. Caspofungin for prophylaxis and treatment of fungal infections in adolescents and adults: a meta-analysis of randomized controlled trials. Pharmazie 2014;67(4):267-73. 